Original Article

\title{
Prescription Pattern of Primary Osteoarthritis in Tertiary Medical Centre
}

\author{
Sow mya Sham Kanneppady ${ }^{1}$, Sham Kishor Kanneppady², Vijaya Raghavan ${ }^{3}$, Aung Myo 00 ${ }^{4}$, Ohn Mar \\ Lwin $^{5}$ \\ ${ }^{1}$ Senior Lecturer and Head, Department of Pharmacology, Faculty of M edicine, Lincoln University College, Selangor Darul \\ Ehsan, M alaysia, ${ }^{2}$ Senior Lecturer, School of Dentistry, International Medical University, Kuala Lumpur, Malaysia, ${ }^{3} \mathrm{Head}$ of \\ the Department of Pharmacology, KVG M edical College and Hospital, Kurunjibag, Sullia, Karnataka, India. \\ ${ }^{4}$ Assistant Professor, Department of Biochemistry, Faculty of M edicine, Lincoln University College, Selangor Darul Ehsan, \\ Malaysia, ${ }^{5}$ Post graduate student, Department of Physiology, Faculty of M edicine, University Malaya, Kuala Lumpur, \\ Malaysia.
}

*Corresponding Author : Sowmya Sham Kanneppady, Senior Lecturer and Head,Department of Pharmacology, Faculty of M edicine, Lincoln University College, No. 2, Jalan Stadium, SS 7/ 15, KelanaJ aya, 47301, PetalingJ aya, Selangor Darul Ehsan, M alaysia.

E-mail : sowmya.sham@gmail.com.

$\begin{array}{ll}\text { Received } & : 12.10 .2017 \\ \text { Review Completed } & : 05.12 .2017 \\ \text { Accepted } & : 06.12 .2017\end{array}$

Keywords: Osteoarthritis, antiinflammatoryagents, prevalence

\begin{tabular}{|c|}
\hline Access this article online \\
\hline Quick Response Code \\
\hline
\end{tabular}

\begin{abstract}
Objectives: Osteoarthritis (OA) is one of the commonest joint/musculoskeletal disorders, affecting the middle aged and elderly, although younger people may be affected as a result of injury or overuse. The study aimed to analyze the data, evaluate the prescription pattern and rationality of the use of drugs in the treatment of primary $\mathrm{OA}$ with due emphasis on the available treatment regimens.
\end{abstract}

Materials and methods: M edical case records of patients suffering from primary $O A$ attending the department of Orthopedics of a tertiary medical centre were the source of data. The study was carried out prospectively for a period of 20 months (from December 2012 to July 2014).

Results: 296 case records were collected in which the total number of drugs prescribed were 550. OA was more common in females (51.7\%) and was more prevalent in the age group of 30-40 years (39\%). Out of 550 drugs prescribed, Aceclofenac was the most frequently prescribed NSAID (29\%) followed by Diclofenac (23\%). Nimesulide and Paracetamol was the most commonly prescribed fixed dose combination (53). Among gastroprotectives, Ranitidine figured in 66 prescriptions. Glucocorticoids were prescribed orally and intraarticularly in 17 and 14 cases respectively. Dietary supplements like Calcium+Vitamin D (42) and Glucosamine Sulfate +Chondroitin Sulfate complex (19) were also prescribed.

Conclusion: The above study highlights the rational use of therapeutic agents for primary $\mathrm{OA}$.

\section{Introduction}

Osteoarthritis (OA) is one of the commonest joint/musculoskeletal disorders, affecting the middle aged and elderly, although younger people may be affected as a result of injury or overuse. $\mathrm{OA}$ is a leading cause of chronic disability of fourth and fifth decades of life [1]. Worldwide estimates are that $9.6 \%$ of men and $18.0 \%$ of women aged over 60 years have symptomatic OA. $80 \%$ of those with osteoarthritis will have limitations in movement, and $25 \%$ cannot perform their major daily activities of life. The incidence is estimated to be 100,000 new cases per year. The risk of mobility impairments caused by knee $\mathrm{OA}$ alone is greater than that due to any other medical condition in people over 65 years [2-4]. Community survey data in rural $\&$ urban areas of India shows the prevalence of osteoarthritis to be in the range of 17 to $60.6 \%$ [5].Among U.S. adults 30 years of age or older, symptomatic disease in the knee occurs in approximately $6 \%$ and symptomatic hip OA in roughly $3 \%$. Since $\mathrm{OA}$ is a disease whose prevalence increases with age, it will become even more prevalent in the future. OA could be primary or secondary and the former is more common. OA can affect any synovial joint, especially weight bearing joints like hip, knee, and spine. Primary OA is also called idiopathic OA; exact cause is not 
known. A genetic basis appears possible. Factors such as obesity, genetics and heredity, occupation involving prolonged standing or squatting can play important roles in primary $O A$.

Disease burden is related to pain occurrence, frequently leading to functional disability ranging from slight limitation of movements to severe impairment of normal daily activities [6]. Therefore, pain relief plays an important role in the treatment of $O A$ Several therapeutic approaches are currently in vogue for the medical management of primary OA. Accordingly, NSAIDs notably piroxicam, diclofenac, aceclofenac, nimesulide, ibuprofen, indomethacin, COX-2 inhibitors and the analgesic paracetamol have been the main therapeutic agents used individually or in approved combinations. Interestingly, other supplementary therapeutic agents such as diacerein, a chondro-modulator, glucosamine sulfate, chondroitin sulfate and/or s- adenosyl methionine have been used to provide functional improvement in patients with $\mathrm{OA}$ and these supplements might provide structure modifying effects that take part in showing the disease progression. Intra articular glucocorticoids have been employed to manage acute joint effusion in patients with knee OA.

In view of the aforementioned observations, this study aims to analyse the data, evaluate the prescription pattern and rationality of the use of drugs in the treatment of primary $\mathrm{OA}$ with due emphasis on the available treatment regimens.

\section{Materials and methods}

A preliminary/pilot study was undertaken in order to gain acquaintance with the topic of the research and collection of data. Accordingly, a special proforma was designed for the collection and compilation of the relevant data for this study. However, with certain need based minor modifications in the said proforma; an initial pilot study was carried out over a period of 2 months to start with, in the department of orthopedics to ascertain feasibility of undertaking such a study. A total of 296 patients suffering from primary $O A$ received treatment over a period of 20 months and the prescriptions (296) for these patients represent the sample size/ material for this study. This study was undertaken with the assistance/coordination of the department of orthopedic surgery. This is essentially a medical audit study comprising the compilation of prospective and observational data and was carried out during the period, December 2012 to July 2014. Patients above the age of 30 years of either gender suffering from primary $\mathrm{OA}$ reporting at a tertiary medical centre were included in the study. Patients < 30 years of age; pregnancy; patients with secondary osteoarthritis (Occurrence of $\mathrm{OA}$ after trauma, infection of the joint) were excluded. The relevant data from the medical case records of the department of orthopedic surgery was accessed by the investigators in person and a special proforma was planned and designed for the collection of data such as:

1. Demographic data: Age, gender, address, OP/IP number and date of examination/admissions of patients

2. Disease data: primary $O A$

3. Data pertaining to drug therapy Drug/drugs prescribed, dose, frequency and duration and route of administration.

4. Incidence of co-morbid disease states, if any.

5. Data pertaining to adverse effects of drug therapy, if any.

The data accessed were subjected to statistical analyses and the relevant statistical methods used were the Pearson chi square, Fisher's exact test and the determination of " $p$ " value.

\section{Results}

296 case records were collected and analyzed This prospective study was conducted in OA patients above the age of 30 years. Primary OA was more commonly reported in the persons of age group 30-40 years (38.9\%) followed by $40-50$ years $(27.4 \%), 50-60$ years $(21.6 \%), 60-70$ years $(7.4 \%)$ and $>70$ years (4.7\%). The mean age group of the patients is 47.49 Primary OA is more common in females (51.7\%) than in males (48.3\%).

As shown in Figure 1 , the site prevalence of primary $\mathrm{OA}$ among the various joints such as left knee (216) followed by right knee (138), left 1st CMC (5), right $1^{\text {st }} \mathrm{CMC}$ (5), left hip 
(1) and right ankle (2).

As shown in Figure 2, Among NSAIDs, Aceclofenac figured in 62 prescriptions followed by diclofenac (49), piroxicam (42). However, naproxen (6) and etoricoxib (4) figured less frequently. Nimesulide and paracetamol (53) was the most commonly prescribed fixed dose combination, followed by Tramadol and Paracetamol (41), Etodolac and paracetamol (8), Aceclofenac and Paracetamol (5), Diclofenac and Serratiopeptidase (4) and Nimesulide and Serratio peptidase (1) in that decreasing order. Among gastroprotectives, $\mathrm{H} 2$ blocker Ranitidine (66) was the most frequently prescribed, followed by the proton pump inhibitors Pantoprazole (49) and Rabeprazole (31) respectively. Among the glucocorticoids, prednisolone was preferred for oral route in 17 prescriptions and triamcinolone (12) and dexamethasone (2) were chosen for intraarticular injections. Diacerein (11) and antidepressant Amitriptyline (9) were also prescribed though rarely. Neutraceuticals-glucosamine sulfate and chondroitin sulfate complex figured in 19 prescriptions. Dietary supplement viz. Calcium+Vitamin D complex was given to 42 patients. Diclofenac was also suggested for intramuscular injection (8) and topical ointment (9).

As showed in Figure 3, the classes of drugs prescribed in order of frequency is NSAIDs (251), gastroprotectives (146), simple analgesic (107), opioids (41), corticosteroids (31), calcium+ vitamin D (42), neutraceuticals (19), diacerein (11) and serratiopeptidase (5).

Out of 296 prescriptions 73 prescriptions were monotherapy, 114 were bitherapy, 81 tritherapy and 28 polytherapy. The pattern for monotherapy in respect of NSAIDs [48], glucocorticoids (20), diacerein (4) and glucosamine + chondroitin sulfate complex (1). In bitherapy (two drug therapy), NSAID and gastroprotectives (44), NSAID and Paracetamol (30) and tramadol and paracetamol (14) prescriptions are noteworthy. The pattern of tritherapy (three drug therapy) and poly therapy (four or more) in various permutations and combinations. Among the routes of administration of the total number of drugs used in 296 prescriptions, with the preferred route being oral (519) followed by injectables (23) and topical (8).

Among the prescription pattern pertaining to NSAIDs, aceclofenac (67), nimesulide (54), diclofenac (53) and piroxicam (42) have been the more frequently prescribed drugs. Table 1 indicates classes of drugs with gender distribution.

Figure 1 : Site prevalence of OA

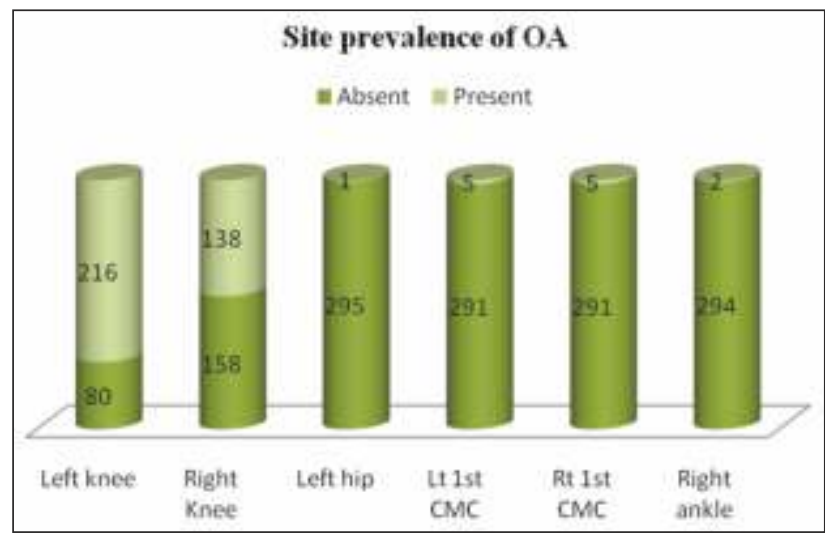

Figure 2 : Overall drug prescription pattern in primary $O A$

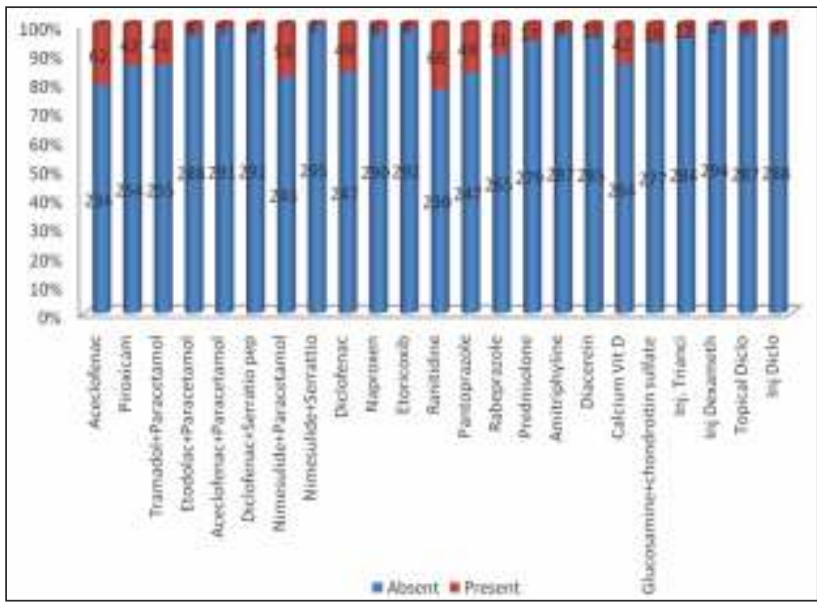

Figure 3 : Classes of drugs prescribed in $\mathrm{OA}$

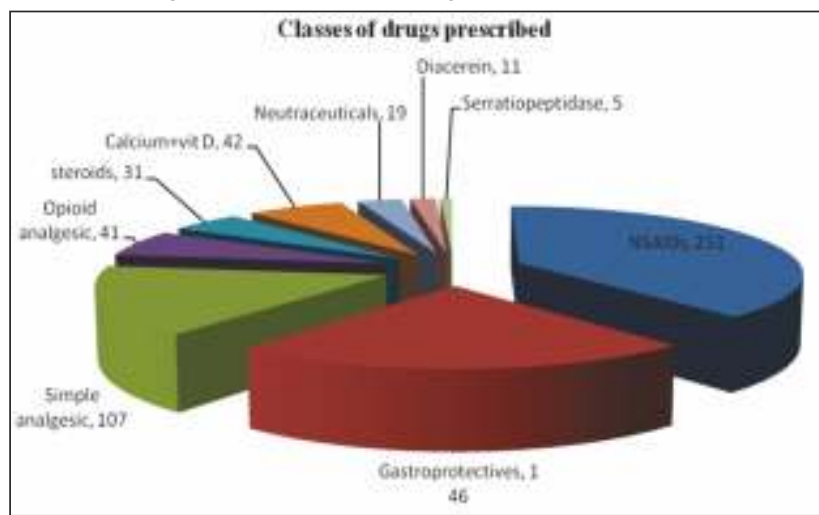


Table 1 : Classes of drugs with gender distribution

\begin{tabular}{|l|c|c|c|c|}
\hline $\begin{array}{l}\text { Classes of drugs } \\
\text { prescribed }\end{array}$ & Males & Females & $\begin{array}{l}\text { Pearson Chi } \\
\text { square test }\end{array}$ & P value \\
\hline NSAIDs & 110 & 124 & 2.884 & 0.410 \\
\hline $\begin{array}{l}\text { Paracetamol } \\
\text { ( Simple analgesic) }\end{array}$ & 52 & 60 & 0.256 & 0.613 \\
\hline Gastroprotectives & 66 & 80 & 1.113 & 0.292 \\
\hline Corticosteroids & 11 & 17 & 1.223 & 0.542 \\
\hline Opioid analgesic & 21 & 20 & 0.161 & 0.688 \\
\hline Amitriptyline & 4 & 5 & 0.056 & 0.814 \\
\hline Diacerein & 5 & 6 & 0.037 & 0.847 \\
\hline Calcium +Vit D & 11 & 31 & 9.591 & 0.002 \\
\hline $\begin{array}{l}\text { Glucosamine } \\
\text { +Chondroitin sulfate }\end{array}$ & 9 & 10 & 0.007 & 0.932 \\
\hline Serratiopeptidase & 1 & 4 & 1.632 & 0.201 \\
\hline
\end{tabular}

\section{Discussion}

This study dwells on the current trends in prescription patterns for the treatment of primary OA. Appropriate data gathered from a total of 296 patients/prescriptions have been considered for medical audit study.

\section{Epidemiological profile}

In this study, the incidence of primary OA has revealed a predilection for the age group 30-40 years (38.9\%) followed by the age group $40-50$ years (27.4\%), 50-60 years (21.6\%), $60-70$ years ( $7.4 \%$ ) and above 70 years (4.7\%). In fact, the occurrence of primary $\mathrm{OA}$ is higher in the younger age group (30-40 years) than in other age groups considered herein, which is noteworthy. This suggested that there is an alarming increase in the younger patients due to changed lifestyle and eating habits [7]. However, gender wise, the incidence of primary $\mathrm{OA}$ is slightly more in females (51.7\%) than males (48.3\%).

\section{Disease data}

Patients afflicted with primary $O A$ as per the inclusion criteria have been chosen for the collection of relevant data and perusal. Accordingly, the various anatomical sites/joints afflicted with primary OA have been noted and these clinical entities are listed. The relative prevalence of primary OA in sites such as knee, hip, Carpometacarpal (CM C) and ankle joints is also highlighted. Interestingly, the knee joints (left and right) have been the most commonly affected sites followed by CMC, ankle and hip joints respectively, in that order, as illustrated in Fig.3.In fact, among the two knee joints, the prevalence of primary $\mathrm{OA}$ has been noted to be higher in the left knee joint than in the right kneejoint.

\section{Data pertaining to drugtherapy}

The prescription patterns observed in this study conform to the oral route of medication mainly and topical and parenteral routes less frequently. Majority of the prescriptions noted herein highlight the oral use of medication with a few exceptions. Several treatment strategies have been suggested for the drug therapy of primary OA. Accordingly, monotherapy (single-drug therapy), bitherapy (two-drug therapy), three-drug therapy and poly therapy (four or more drug therapy) have been in vogue.

The detailed drug data in the treatment of primary $\mathrm{OA}$ is as follows:

Several classes of drugs have been used for affording therapeutic benefit in primary $\mathrm{OA}$ with the particular drug being chosen, either singly or in approved combinations with or without adjuncts.

Incidentally, NSAIDs, gastroprotectives, paracetamol, calcium+vit D complex, opioid analgesic (tramadol), glucocorticoids, diacerein, neutraceuticals and serratiopeptidase represent the various classes of drugs prescribed. Aceclofenac, piroxicam, diclofenac, etoricoxib, naproxen, etodolac and nimesulide are the commonly chosen NSAIDs and one of these is used in the form of monotherapy or polytherapy with suitable adjuncts. Gastroprotectives, calcium+vit D complex, diacerein, neutraceuticals and serratiopeptidase constitute the common adjuncts/supplementary agents used singly or in various approved permutations and combinations, with an NSAID, most often. Interestingly, paracetamol has been an important drug chosen for combination with an opioid analgesic viz. tramadol. Diacerein or the combination of glucosamine sulfate and chondroitic sulfate is prescribed as an adjunct for improving the functional capacity of the affected joints. Intraarticular/oral glucocorticoids (viz. triamcinolone or prednisolone) has also been suggested 
for the treatment of primary OA either alone or with one of the NSAIDs.

\section{Strategies of drugtherapies/ prescriptions}

Monotherapy: NSAIDs have been the mainstay and outnumber the other classes of drugs employed for monotherapy, when compared with paracetamol, glucocorticoids, diacerein and the complex of glucosamine sulfate +chondroitin sulfate. Infact, monotherapy is noted in 73 out of 296 prescriptions.

Bitherapy: A total of 114 prescriptions constitute bitherapy and among these, the combinations of an NSAID with a gastroprotective (38.5\%), an NSAID with paracetamol (26.3\%) have been the preferred ones. The prescriptions comprising combination of an opioid analgesic (tramadol) with paracetamol are also noteworthy though less frequent. The rare/infrequent use of amitriptyline is also indicated.

Tritherapy therapy: A total of 81 prescriptions reveal the use of three drugs concurrently and here also NSAIDs have been the preferential drugs prescribed in different permutations and combinations with supplementary agents. In fact, opioids, paracetamol, gastroprotectives and neutraceuticals represent the important accompaniments in the various treatment strategies. Incidentally, the combination of opioid with paracetamol along with gastroprotectives has also been an important therapeutic regimen (in $14.8 \%$ of three drug therapy). However, the use of amitriptyline and oral/intraarticular glucocorticoids is rarely recommended, as is diacerein/glucosamine/chondroitin sulfate. Serratiopeptidase has also figured in the list of useful adjuncts, though less frequently. Similar results were obtained in study conducted by Sharma Tet.al [8].

Poly drug therapy: In this four or more drugs have been

\section{References}

1. Kulkarni C, Leena A, Lohit K. A randomised comparative study safety and efficacy of immediate release glucosamine $\mathrm{HCL}$ with Glucosamine HCL sustained release formulation in the treatment of knee osteoarthritis: A proof of concept study. J Pharmacol pharmacother 2012; 3:48-54.

2. Egloff C, Hügle T, Valderrabano V. Biomechanics and pathomechanisms of osteoarthritis. Swiss Med Wkly. 2012 prescribed concurrently to confer the desired therapeutic benefit. A total of 28 prescriptions illustrate the various treatment regimens comprising the main therapeutic agents such as NSAIDs in combination with well known adjuncts. Interestingly, the concurrent administration of an NSAID, paracetamol, a gastroprotective and a nutritional supplement viz. calcium + vit $D$, has been the preferred / prominent four drug therapy (in $50 \%$ of polytherapy), possibly to achieve synergistic benefit. Other adjuncts/ supplementary drugs have also been employed in different permutations and combinations.

\section{Routes of drug administration}

In general, oral route has been the preferred one in the majority of prescriptions (94.36\%) followed by the injectable route $(4.18 \%)$ and the topical route $(1.45 \%)$, in that order. It is worthwhile noting that for the purpose of description in this study, NSAIDs, paracetamol, opioids and glucocorticoids have been regarded as the primary therapeutic agents whereas the others such as gastroprotectives, serratiopeptidase, diacerein, glucosamine+ chondroitin sulfate complex, calcium+vit D complex have been considered as adjuncts/supplementary agents or secondary therapeutic agents.

\section{Conclusion}

In a nutshell, a wide variety of therapeutic agents are in vogue for the drug therapy of primary OA. Among the NSAIDs, Aceclofenac (67), nimesulide (54), diclofenac (53) and piroxicam (42) have been the more frequently prescribed drugs.

Modest prescribing practices are evident in the hospital where this study was undertaken and the prescription patterns are in consensus with the general trends in vogue, with a few changes, probably in the choice of established primary and supplementary therapeutic agents.

142:w13583.

3. Prevalence and Impact of chronic joint symptoms - seven states. M M WR - M orb M ortal Wkly Rep. 1998; 47:345-351.

4. Guccione AA, Felson DT, Anderson JJ, Anthony JM , Zhang Y, Wilson PW. The effects of specific medical conditions on the functional limitations of elders in the Framingham Study. Am J Public Health. 1994; $84: 351-358$ 
5. Sharma MK, Swami HM, Bhatia V, Verma A, Bhatia S, Kaur G. An epidemiological study of correlates of osteo-arthritis in geriatric population of UT Chandigarh. Indian J Community M ed 2007; 32:7778.

6. Alacqua M, Trifiro G, Cavagna L, Caporali R, Montecucco CM. Prescribing pattern of drugs in the treatment of Osteoarthritis in Italian general practice: The effect of rofecoxib withdrawal. Arthritis Care and Research 2008; 59:568-574.
7. Bishnoi M, Kumar A, Kulkarni SK. Prescription pattern monitoring of management of pattern of osteoarthritis non steroidal anti inflammatory drugs at PUHC, Chandigarh. Indian J. Pharm. Sci., 2006; 68:525-527.

8. Sharma T, Dutta S, Dhasmana DC. Prescribing pattern of NSAIDs in orthopaedic OPD of a tertiary care teaching hospital in Uttaranchal. JK Sci 2006; 8:160-162. 